\title{
A QUASI-MONTE CARLO METHOD FOR THE BOLTZMANN EQUATION
}

\author{
CHRISTIAN LECOT
}

\begin{abstract}
A new quasi-Monte Carlo method for solving the Boltzmann equation in a simplified case is described. The analysis is restricted to a spatially homogeneous and isotropic gas; in addition, the molecular model only involves isotropic scattering. The scheme makes use of particles and combines an Euler scheme with numerical integrations. The sequence which is used for the quadratures must possess some symmetry properties which prescribe energy conservation for colliding particles. The error of the method is estimated by means of the discrepancy of the sequence which performs the quadratures. An algorithm for generating convenient sequences is proposed. In an example, where an exact solution is known, the computation of effective errors is included.
\end{abstract}

\section{INTRODUCTION}

Rarefied gas flows are usually simulated by Monte Carlo techniques. Besides the successful Direct Simulation Monte Carlo (DSMC) method of Bird [2], another scheme was derived by Nanbu [14] from the Boltzmann equation itself. A drawback of both schemes are numerical fluctuations caused by the use of pseudorandom numbers. An improved Monte Carlo scheme, which reduces fluctuations, has recently been developed at the University of Kaiserslautern [1]: it will be referred to as the KMC scheme.

A fully deterministic method for solving the Boltzmann equation is proposed here. The constraints on the analysis are the following. We consider an infinite spatially homogeneous and isotropic gas (the velocity distribution is radially symmetric). The molecular model is characterized by isotropic scattering (the differential cross section $\sigma$ depends only on the relative speed $g$ and not on the deflection angle). In addition, we assume that $g \sigma(g)$ is some nonnegative, nondecreasing, and bounded function. The hypothesis on the cross section allows physically relevant models, such as the VHS model of Bird [3], when a cutoff is used. In earlier communications $[10,11]$ the simplest choice $(g \sigma(g)$ equals a constant) was considered. A deterministic version of the scheme of Nanbu, that we called the Low Discrepancy (LD) method, was described. An error analysis was proposed and it was shown that the LD method outperforms the original scheme of Nanbu in a test case where an exact solution is known.

Received March 1, 1990.

1980 Mathematics Subject Classification (1985 Revision). Primary 65M15; Secondary 65C05, $11 \mathrm{~K} 38$. 
The scheme of the present paper will be called the Quasi-Monte Carlo (QMC) method. The QMC method (as well as the scheme of Nanbu or the LD method) can be summarized as follows. The initial velocity distribution $v^{2} f_{0}(v) d v$ (in $\mathbf{R}_{+}$) is approximated by a sum of $N$ Dirac measures

$$
f^{(0)}=\frac{1}{N} \sum_{i=1}^{N} \delta\left(v-v_{i}^{(0)}\right)
$$

The time is discretized by steps of length $\Delta t$; the approximation

$$
f^{(n)}=\frac{1}{N} \sum_{i=1}^{N} \delta\left(v-v_{i}^{(n)}\right)
$$

of the velocity distribution at time $t^{(n)}=n \Delta t$ is obtained from $f^{(n-1)}$ in two steps. First, a measure $g^{(n-1)}$ is generated by an Euler (forward difference) scheme. Then $f^{(n)}$ is obtained from $g^{(n-1)}$ by a quasi-Monte Carlo integration. The $v_{i}^{(n)}, 1 \leq i \leq N$, are referred to as the velocities of simulated particles (or molecules) at time $t^{(n)}$. The QMC method is different from the scheme of Nanbu but is similar to the DSMC scheme of Bird in two ways. First, the scheme is based on binary collisions, and energy conservation is prescribed for colliding particles (for the procedure of Nanbu, as well as for the LD method, the total energy changes at every time step). This is achieved by using a symmetrical sequence for the quasi-Monte Carlo integration. Second, the computing task is proportional to $N \log N$, where $N$ is the number of simulated molecules (the computing task of the algorithm of Nanbu is proportional to $N^{2}$, unless the molecular model is Maxwellian and the LD scheme only handles a simplified Maxwellian model).

An error analysis of the QMC method is included in the paper. An essential tool is the concept of discrepancy, which was also used for the numerical analysis of the LD method. For $P$ points $\mathbf{x}_{1}, \ldots, \mathbf{x}_{P}$ in the $s$-dimensional unit cube $I^{s}=[0,1)^{s}, s \geq 1$, the discrepancy (relative to the Lebesgue measure) is defined by

$$
D_{P}(\mathbf{X})=\sup _{J}\left|\frac{A(J, \mathbf{X})}{P}-\right| J||,
$$

where $J$ runs through all subintervals of $I^{s}, A(J, \mathbf{X})$ is the number of $p, 1 \leq$ $p \leq P$, with $\mathbf{x}_{p} \in J$, and $|J|$ is the measure of $J$. The *-discrepancy of the $P$ points $\mathbf{x}_{1}, \ldots, \mathbf{x}_{P}$ is defined by

$$
D_{P}^{*}(\mathbf{X})=\sup _{J}\left|\frac{A(J, \mathbf{X})}{P}-\right| J||,
$$

where $J$ runs through all subintervals of $I^{S}$ containing $0 . D_{P}(\mathbf{X})$ and $D_{P}^{*}(\mathbf{X})$ are linked by

$$
D_{P}^{*}(\mathbf{X}) \leq D_{P}(\mathbf{X}) \leq 2^{s} D_{P}^{*}(\mathbf{X}) .
$$

For an infinite sequence $\mathbf{x}_{1}, \mathbf{x}_{2}, \ldots$ of points in $I^{S}$ we define $D_{P}(\mathbf{X})$, resp. $D_{P}^{*}(\mathbf{X})$, to be the discrepancy, resp. *-discrepancy, of the first $P$ terms of 
the sequence. We refer to $[9,16]$ for further information on the concept of discrepancy. We will measure the error at time $t^{(n)}$ of the QMC method by the $*$-discrepancy of $v_{1}^{(n)}, \ldots, v_{N}^{(n)}$ relative to the exact velocity distribution at time $t^{(n)}$. If the discrete velocities are ordered according to their magnitude, the error of the scheme can be estimated by means of the discrepancy of the sequence used for the quasi-Monte Carlo integration.

The construction of the sequence that performs the quadratures is based on the recent theory of $(0, s)$-sequences [17]. We propose in this paper an algorithm for generating $(0, s)$-sequences which is suited to vector computers. A convenient symmetrical sequence is obtained from a $(0, s)$-sequence by reflection. Its $*$-discrepancy is bounded by means of the $*$-discrepancy of the $(0, s)$-sequence which is used. When $g \sigma(g)$ equals some constant, an exact solution of the Boltzmann equation for a specific initial velocity distribution was discovered by Krook and Wu [8]. The effective error of the QMC method at a given time $T$ can be computed in this case.

The paper is organized as follows. In $\S 1$ the QMC method is presented and the assumptions on the sequence used for the quadratures are given. In $\S 2$ some error estimates are demonstrated. They are derived from error bounds for quasi-Monte Carlo integration. In $\S 3$ the sequence used for the quadratures is constructed, its $*$-discrepancy is estimated, and effective errors in the case considered by Krook and $\mathrm{Wu}$ are computed. They are compared with errors of the KMC scheme.

\section{THE QMC METHOD}

We present the Boltzmann equation for a spatially homogeneous and isotropic gas. We introduce the weak formulation, which is used for determining an approximation to the solution. We also discuss the assumptions on the cross section. We refer to $[4,18]$ for the derivation of the Boltzmann equation and for related concepts; the derivation of the weak formulation was given in an earlier communication [10].

Let $f_{0}$ be a positive function on $\mathbf{R}_{+}$such that

$$
\int_{\mathbf{R}_{+}} v^{2} f_{0}(v) d v=1
$$

Let $f$ be a regular positive function on $\mathbf{R}_{+}^{2}$ which satisfies the Boltzmann equation

$$
\begin{aligned}
\frac{\partial f}{\partial t}(|\mathbf{v}|, t)= & \frac{n^{*}}{4 \pi} \int_{\mathbf{R}^{3} \times S_{\mathbf{v}, \mathbf{w}}^{2}}\left(f\left(\left|\mathbf{v}^{\prime}\right|, t\right) f\left(\left|\mathbf{w}^{\prime}\right|, t\right)-f(|\mathbf{v}|, t) f(|\mathbf{w}|, t)\right) \\
& \cdot|\mathbf{v}-\mathbf{w}| s(\theta,|\mathbf{v}-\mathbf{w}|) d \mathbf{w} d \mathbf{n}, \quad \mathbf{v} \in \mathbf{R}^{3}, t \in \mathbf{R}_{+},
\end{aligned}
$$

where $|\cdot|$ is the Euclidean norm, $n^{*}$ is the number of molecules per unit volume, $S_{\mathbf{v}, \mathbf{w}}^{2}=\left\{\mathbf{n} \in \mathbf{R}^{3}:|\mathbf{n}|=1, \mathbf{n} \cdot(\mathbf{v}-\mathbf{w})>0\right\}, \mathbf{v}^{\prime}=\mathbf{v}-\mathbf{n} \cdot(\mathbf{v}-\mathbf{w}) \mathbf{n}, \mathbf{w}^{\prime}=$ $\mathbf{w}+\mathbf{n} \cdot(\mathbf{v}-\mathbf{w}) \mathbf{n}, s$ is the differential scattering cross section, and $\theta$ is the angle 
between $\mathbf{n}$ and $\mathbf{v}-\mathbf{w}$; with the initial condition

$$
f(|\mathbf{v}|, 0)=f_{0}(|\mathbf{v}|), \quad \mathbf{v} \in \mathbf{R}^{3} .
$$

If $B\left(\mathbf{R}_{+}\right)$is the space of all bounded measurable functions everywhere defined on $\mathbf{R}_{+}, f$ also satisfies

$$
\begin{aligned}
& \frac{d}{d t} \int_{\mathbf{R}_{+}} \varphi(v) v^{2} f(v, t) d v \\
& =\frac{n^{*}}{(4 \pi)^{2}} \int_{\mathbf{R}^{6} \times S^{2}}\left(\varphi\left(\mathbf{v}^{\prime} \mid\right)-\varphi(|\mathbf{v}|)\right)|\mathbf{v}-\mathbf{w}| \sigma(\chi,|\mathbf{v}-\mathbf{w}|) \\
& \quad \cdot f(|\mathbf{v}|, t) f(|\mathbf{w}|, t) d \mathbf{v} d \mathbf{w} d \nu, \quad \varphi \in B\left(\mathbf{R}_{+}\right), \quad t \in \mathbf{R}_{+},
\end{aligned}
$$

where

$$
\begin{gathered}
\mathbf{v}^{\prime}=\frac{1}{2}(\mathbf{v}+\mathbf{w}+|\mathbf{v}-\mathbf{w}| \nu), \\
\chi=\pi-2 \theta \text { is the deflection angle, } \\
\nu=\frac{1}{|\mathbf{v}-\mathbf{w}|}(\mathbf{v}-\mathbf{w}-2 \mathbf{n} \cdot(\mathbf{v}-\mathbf{w}) \mathbf{n}) .
\end{gathered}
$$

The cross section $\sigma$ is related to $s$ by $s(\theta, g)=4 \cos \theta \sigma(\chi, g), \theta \in\left(0, \frac{\pi}{2}\right)$, $g \in \mathbf{R}_{+}$.

We only consider molecular models characterized by isotropic scattering, i.e., $\sigma$ does not depend on $\chi$. This restriction drastically reduces the computation time per collision when a numerical procedure is used. Moreover, Bird [3] has shown that "requirements for the accurate modelling of engineering flows are best met by a molecular model called the Variable Hard Sphere (VHS) model", which involves isotropic scattering. In the VHS model the total collision cross section $\sigma_{T}=4 \pi \sigma$ is related to the relative velocity $g$ by

$$
\sigma_{T}(g)=\sigma_{\text {ref }}\left(\frac{4(\eta-2)}{\eta-1} \frac{k T_{\text {ref }}}{m_{r}}\right)^{2 /(\eta-1)} g^{-4 /(\eta-1)}, \quad g \in \mathbf{R}_{+},
$$

where $\sigma_{\text {ref }}$ is a reference cross section calculated at temperature $T_{\text {ref }}, \eta$ is the exponent of the inverse power law molecular force, $k$ is the Boltzmann constant, and $m_{r}$ is the reduced mass.

The exponent $\eta$ lies between 5 and $\infty \quad(\eta=5$ for the Maxwell model and $\eta=\infty$ for the hard sphere model).

It is convenient at this point to introduce a new function $q$ defined by

$$
q(g)=n^{*} g \sigma_{T}(g), \quad g \in \mathbf{R}_{+} .
$$

We assume

$q$ is a positive monotonic function,

$$
Q=\sup _{g \in \mathbf{R}_{+}} q(g)<+\infty .
$$


Condition (1.7) is satisfied by the VHS model. Condition $\left(1.7^{\prime}\right)$ means that $\sigma_{T}$ must be truncated (in the case of the VHS model). This hypothesis is reasonable if the high-energy part of the velocity distribution is underpopulated. In earlier communications $[10,11], q(g)=4 k \pi$ for some constant $k>0$.

The right-hand side of $(1.4)$ is now expressed by using the variables

$$
v=|\mathbf{v}|, \quad w=|\mathbf{w}|, \quad x^{(4)}=\frac{1}{2}\left(1+\frac{\mathbf{v} \cdot \mathbf{w}}{|\mathbf{v}||\mathbf{w}|}\right), \quad x^{(5)}=\frac{1}{2}\left(1+\frac{(\mathbf{v}+\mathbf{w}) \cdot \nu}{|\mathbf{v}+\mathbf{w}|}\right) .
$$

For $\left(v, w, x^{(4)}, x^{(5)}\right) \in \mathbf{R}_{+}^{2} \times I^{2}$ let

$$
\begin{gathered}
{\left[v, w ; x^{(4)} ; x^{(5)}\right]} \\
=\frac{\left(v^{2}+w^{2}+\left(\left(v^{2}+w^{2}\right)^{2}-4\left(2 x^{(4)}-1\right)^{2} v^{2} w^{2}\right)^{1 / 2}\left(2 x^{(5)}-1\right)\right)^{1 / 2}}{2^{1 / 2}}, \\
{\left[v, w ; x^{(4)}\right]=\left(v^{2}+w^{2}-2\left(2 x^{(4)}-1\right) v w\right)^{1 / 2} .}
\end{gathered}
$$

We then obtain the convenient weak formulation

$$
\begin{aligned}
& \frac{d}{d t} \int_{\mathbf{R}_{+}} \varphi(v) v^{2} f(v, t) d v \\
& =\int_{\mathbf{R}_{+}^{2} \times I^{2}}\left(\varphi\left(v^{\prime}\right)-\varphi(v)\right) q(g) v^{2} f(v, t) \\
& \quad \cdot w^{2} f(w, t) d v d w d x^{(4)} d x^{(5)}, \quad \varphi \in B\left(\mathbf{R}_{+}\right), t \in \mathbf{R}_{+},
\end{aligned}
$$

where

$$
v^{\prime}=\left[v, w ; x^{(4)} ; x^{(5)}\right], \quad g=\left[v, w ; x^{(4)}\right] .
$$

Starting from (1.10), we present the QMC method. It combines an Euler scheme (step (i) and equation (1.14)) with quasi-Monte Carlo integration (step (ii) and equation (1.17)). The numerical procedure is linked with molecular gas dynamics by two conditions on the sequence used for the quadratures.

We denote by $\delta\left(v-v_{0}\right)$ the Dirac measure located at the point $v_{0} \in \mathbf{R}$. An integer $N>0$ defines the accuracy of the approximation. A time step $\Delta t$ is chosen such that

$$
\Delta t Q \leq 1
$$

This condition ensures the feasibility of the scheme. We also need a sequence

$$
\mathbf{X}=\left\{\mathbf{x}_{n}: n \geq 1\right\} \subset I^{5}
$$

for quasi-Monte Carlo integration. We introduce discrete times $t^{(n)}=n \Delta t$, $n \geq 0$, and sets

$$
\mathbf{X}^{(n)}=\left\{\mathbf{x}_{p}: n N<p \leq(n+1) N\right\}, \quad n \geq 0 .
$$

A set $V^{(0)}=\left\{v_{i}^{(0)}: 1 \leq i \leq N\right\} \subset \mathbf{R}_{+}$is chosen such that

$$
f^{(0)}=\frac{1}{N} \sum_{i=1}^{N} \delta\left(v-v_{i}^{(0)}\right)
$$


approximates (in a sense that will be subsequently specified) the initial velocity distribution $v^{2} f_{0}(v) d v$.

For $n \geq 0$ we describe the scheme to compute

$$
V^{(n+1)}=\left\{v_{i}^{(n+1)}: 1 \leq i \leq N\right\} \subset \mathbf{R}_{+}
$$

and

$$
f^{(n+1)}=\frac{1}{N} \sum_{i=1}^{N} \delta\left(v-v_{i}^{(n+1)}\right)
$$

(which approximates $v^{2} f\left(v, t^{(n+1)}\right) d v$ ) if $V^{(n)}$ is known.

(i) A measure $g^{(n)}$ on $\mathbf{R}_{+}$is defined by

$$
\begin{array}{r}
\int_{\mathbf{R}_{+}} \varphi(v) g^{(n)}(d v)=\int_{\mathbf{R}_{+}} \varphi(v) f^{(n)}(d v)+\Delta t \int_{\mathbf{R}_{+}^{2} \times I^{2}}\left(\varphi\left(v^{\prime}\right)-\varphi(v)\right) q(g) \\
\cdot f^{(n)}(d v) f^{(n)}(d w) d x^{(4)} d x^{(5)}, \quad \varphi \in B\left(\mathbf{R}_{+}\right),
\end{array}
$$

or, equivalently,

$$
\begin{aligned}
\int_{\mathbf{R}_{+}} \varphi(v) g^{(n)}(d v) & \\
= & \frac{1}{N} \sum_{i=1}^{N}\left(1-\frac{\Delta t}{N} \sum_{j=1}^{N} \int_{I} q\left(\left[v_{i}^{(n)}, v_{j}^{(n)} ; x^{(4)}\right]\right) d x^{(4)}\right) \varphi\left(v_{i}^{(n)}\right) \\
+ & \frac{\Delta t}{N^{2}} \sum_{i=1}^{N} \sum_{j=1}^{N} \int_{I^{2}} q\left(\left[v_{i}^{(n)}, v_{j}^{(n)} ; x^{(4)}\right]\right) \\
& \cdot \varphi\left(\left[v_{i}^{(n)}, v_{j}^{(n)} ; x^{(4)}, x^{(5)}\right]\right) d x^{(4)} d x^{(5)}, \quad \varphi \in B\left(\mathbf{R}_{+}\right) .
\end{aligned}
$$

(ii) For $1 \leq i \leq N$ and $1 \leq j \leq N$ let $\chi_{i, j}$ be the characteristic function of $\left[\frac{i-1}{N}, \frac{i}{N}\right) \times\left[\frac{j-1}{N}, \frac{j}{N}\right)$, and let $c_{i, j}^{(n)}$ be the characteristic function of $\left\{\left(x^{(3)}, x^{(4)}\right) \in I^{2}: x^{(3)}<\Delta t q\left(\left[v_{i}^{(n)}, v_{j}^{(n)} ; x^{(4)}\right]\right)\right\}$. If $L^{(n)} \varphi$ is defined by

$$
\begin{array}{r}
L^{(n)} \varphi(\mathbf{x})=\sum_{i=1}^{N} \sum_{j=1}^{N} \chi_{i, j}\left(x^{(1)}, x^{(2)}\right)\left(\left(1-c_{i, j}^{(n)}\left(x^{(3)}, x^{(4)}\right)\right) \varphi\left(v_{i}^{(n)}\right)\right. \\
\left.+c_{i, j}^{(n)}\left(x^{(3)}, x^{(4)}\right) \varphi\left(\left[v_{i}^{(n)}, v_{j}^{(n)} ; x^{(4)}, x^{(5)}\right]\right)\right),
\end{array}
$$

for $\mathbf{x} \in I^{5}$, then

$$
\int_{I^{5}} L^{(n)} \varphi(\mathbf{x}) d \mathbf{x}=\int_{\mathbf{R}_{+}} \varphi(v) g^{(n)}(d v), \quad \varphi \in B\left(\mathbf{R}_{+}\right) .
$$

Now define $f^{(n+1)}$ by

$$
\int_{\mathbf{R}_{+}} \varphi(v) f^{(n+1)}(d v)=\frac{1}{N} \sum_{l=1}^{N} L^{(n)} \varphi\left(\mathbf{x}_{n N+l}\right), \quad \varphi \in B\left(\mathbf{R}_{+}\right) .
$$


We denote by $\lfloor z\rfloor$ the greatest integer $\leq z$. For a fixed $n$ let

$$
i(l)=1+\left\lfloor N x_{n N+l}^{(1)}\right\rfloor, \quad j(l)=1+\left\lfloor N x_{n N+l}^{(2)}\right\rfloor, \quad 1 \leq l \leq N .
$$

If

$$
\mathbf{x}=\left(x^{(1)}, x^{(2)}, x^{(3)}, x^{(4)}, x^{(5)}\right) \in I^{5}
$$

we set

$$
\mathbf{x}^{*}=\left(x^{(2)}, x^{(1)}, x^{(3)}, x^{(4)}, 1-x^{(5)}\right) .
$$

Each molecule collides at most once within the time interval $\left[t^{(n)}, t^{(n+1)}\right)$, and energy is conserved at each collision provided two conditions are satisfied:

$$
\begin{gathered}
i(l) \text { and } j(l) \text { are one-to-one mappings } \\
\text { of the set }\{1, \ldots, N\} \text { onto itself, }
\end{gathered}
$$

$$
\text { if } \mathbf{x} \in \mathbf{X}^{(n)} \text {, then } \mathbf{x}^{*} \in \mathbf{X}^{(n)} \text {. }
$$

No condition is required for momentum conservation because the momentum density of a spatially homogeneous and isotropic gas equals 0 and is automatically conserved. The velocities of the molecules at time $t^{(n+1)}$ are then conveniently expressed.

If

$$
x_{n N+l}^{(3)}<\Delta t q\left(\left[v_{i(l)}^{(n)}, v_{j(l)}^{(n)} ; x_{n N+l}^{(4)}\right]\right),
$$

then molecules $i(l)$ and $j(l)$ collide and the new velocities are

$$
\begin{aligned}
& v_{i(l)}^{(n+1)}=\left[v_{i(l)}^{(n)}, v_{j(l)}^{(n)} ; x_{n N+l}^{(4)}, x_{n N+l}^{(5)}\right], \\
& v_{j(l)}^{(n+1)}=\left[v_{j(l)}^{(n)}, v_{i(l)}^{(n)} ; x_{n N+l}^{(4)}, 1-x_{n N+l}^{(5)}\right] .
\end{aligned}
$$

If

$$
x_{n N+l}^{(3)} \geq \Delta t q\left(\left[v_{i(l)}^{(n)}, v_{j(l)}^{(n)} ; x_{n N+l}^{(4)}\right]\right),
$$

then molecules $i(l)$ and $j(l)$ do not collide and

$$
v_{i(l)}^{(n+1)}=v_{i(l)}^{(n)}, \quad v_{j(l)}^{(n+1)}=v_{j(l)}^{(n)} \text {. }
$$

An additional computational step will permit us to estimate the error of the QMC method:

$$
\text { each set } V^{(n)} \text { is ordered such that if } i \leq j \text {, then } v_{i}^{(n)} \leq v_{j}^{(n)} \text {. }
$$

\section{ERROR ESTIMATES}

We define the error at time $t^{(n)}$ of the QMC method and we introduce some error terms. An estimate of the first error term (Lemma 2.1) is derived from an error estimate used in quasi-Monte Carlo integration. The second error term is easily bounded (Lemma 2.2). 
If $r>0$, we denote by $\varphi_{r}$ the characteristic function of $[0, r)$. We subsequently use the following result. If $\left(r, w, x^{(4)}, x^{(5)}\right) \in \mathbf{R}_{+}^{2} \times I^{2}$, there exists $s\left(r, w, x^{(4)}, x^{(5)}\right) \in[0, \infty]$ such that

$$
\varphi_{r}\left(\left[v, w ; x^{(4)}, x^{(5)}\right]\right)=\varphi_{s\left(r, w, x^{(4)}, x^{(5)}\right)}(v), \quad v \in \mathbf{R}_{+} .
$$

For $n \geq 0$ we set

$$
d_{N}^{(n)}(r)=\frac{1}{N} \sum_{i=1}^{N} \varphi_{r}\left(v_{i}^{(n)}\right)-\int_{\mathbf{R}_{+}} \varphi_{r}(v) v^{2} f\left(v, t^{(n)}\right) d v, \quad r>0,
$$

and we measure the error at time $t^{(n)}$ of the QMC method by

$$
D_{N}^{*}\left(V^{(n)}, v^{2} f_{n}\right)=\sup _{r>0}\left|d_{N}^{(n)}(r)\right| .
$$

According to the definition of Hlawka and Mück [7], $D_{N}^{*}\left(V^{(n)}, v^{2} f_{n}\right)$ is the *-discrepancy of $V^{(n)}$ relative to $v^{2} f\left(v, t^{(n)}\right) d v$. We need some additional error terms:

$$
\begin{aligned}
e_{N}^{(n)}(r)= & \int_{\mathbf{R}_{+}^{2} \times I^{2}}\left(\varphi_{r}\left(v^{\prime}\right)-\varphi_{r}(v)\right) q(g) f^{(n)}(d v) f^{(n)}(d w) d x^{(4)} d x^{(5)} \\
& -\int_{\mathbf{R}_{+}^{2} \times I^{2}}\left(\varphi_{r}\left(v^{\prime}\right)-\varphi_{r}(v)\right) q(g) v^{2} f\left(v, t^{(n)}\right) \\
& \cdot w^{2} f\left(w, t^{(n)}\right) d v d w d x^{(4)} d x^{(5)}
\end{aligned}
$$

where $v^{\prime}$ and $g$ are given by (1.11),

$$
\begin{gathered}
\varepsilon^{(n)}(r)=\int_{\left(t^{(n)}, t^{(n+1)}\right) \times \mathbf{R}_{+}} \varphi_{r}(v) v^{2}\left(\frac{\partial f}{\partial t}\left(v, t^{(n)}\right)-\frac{\partial f}{\partial t}(v, t)\right) d v d t \\
\delta_{N}^{(n)}(r)=\frac{1}{N} \sum_{l=1}^{N} L^{(n)} \varphi_{r}\left(\mathbf{x}_{n N+l}\right)-\int_{I^{5}} L^{(n)} \varphi_{r}(\mathbf{x}) d \mathbf{x} \\
\Delta_{N}^{(n)}(r)=\sum_{m=0}^{n-1} \delta_{N}^{(m)}(r)
\end{gathered}
$$

The various error terms are linked by the relation

$$
d_{N}^{(n)}(r)=d_{N}^{(n-1)}(r)+\Delta t e_{N}^{(n-1)}(r)+\varepsilon^{(n-1)}(r)+\delta_{N}^{(n-1)}(r), \quad n \geq 1 .
$$

Consequently,

$$
d_{N}^{(n)}(r)=d_{N}^{(0)}(r)+\Delta t \sum_{m=0}^{n-1} e_{N}^{(m)}(r)+\sum_{m=0}^{n-1} \varepsilon^{(m)}(r)+\Delta_{N}^{(n)}(r), \quad n \geq 1 .
$$

An estimate of the error term $e_{N}^{(n)}(r)$ is obtained by using the following inequality, which is due to Koksma (we refer to $[9,16]$ ). 
If $g$ is a function of bounded variation $V(g)$ on $I$ and $x_{1}, \ldots, x_{P}$ are numbers in $I$ with *-discrepancy $D_{P}^{*}(X)$, then

$$
\left|\frac{1}{P} \sum_{i=1}^{P} g\left(x_{i}\right)-\int_{0}^{1} g(x) d x\right| \leq V(g) D_{P}^{*}(X) .
$$

Lemma 2.1. The error term (2.4) can be estimated by

$$
\left|e_{N}^{(n)}(r)\right| \leq 11 Q D_{N}^{*}\left(V^{(n)}, v^{2} f_{n}\right) .
$$

Proof. The error term $e_{N}^{(n)}(r)$ is split into sums or integrals where the following differences appear:

$$
\begin{gathered}
\frac{1}{N} \sum_{i=1}^{N} q\left(\left[v_{i}^{(n)}, w ; x^{(4)}\right]\right)-\int_{\mathbf{R}_{+}} q\left(\left[v, w ; x^{(4)}\right]\right) v^{2} f\left(v, t^{(n)}\right) d v \\
\frac{1}{N} \sum_{i=1}^{N} \varphi_{r}\left(v_{i}^{(n)}\right) q\left(\left[v_{i}^{(n)}, w ; x^{(4)}\right]\right)-\int_{\mathbf{R}_{+}} \varphi_{r}(v) q\left(\left[v, w ; x^{(4)}\right]\right) v^{2} f\left(v, t^{(n)}\right) d v
\end{gathered}
$$

and

$$
\begin{aligned}
& \frac{1}{N} \sum_{i=1}^{N} \varphi_{r}\left(\left[v_{i}^{(n)}, w ; x^{(4)}, x^{(5)}\right]\right) q\left(\left[v_{i}^{(n)}, w ; x^{(4)}\right]\right) \\
& \quad-\int_{\mathbf{R}_{+}} \varphi_{r}\left(\left[v, w ; x^{(4)}, x^{(5)}\right]\right) q\left(\left[v, w ; x^{(4)}\right]\right) v^{2} f\left(v, t^{(n)}\right) d v .
\end{aligned}
$$

They are estimated by using (2.1), (2.10), and some techniques of Hlawka and Mück [7].

We add for the sake of completeness the following minor result.

Lemma 2.2. The error term (2.5) satisfies the inequality

$$
\left|\varepsilon^{(n)}(r)\right| \leq \Delta t \int_{\left(t^{(n)}, t^{(n+1)}\right) \times \mathbf{R}_{+}} v^{2}\left|\frac{\partial^{2} f}{\partial t^{2}}(v, t)\right| d v d t .
$$

The third error term $\Delta_{N}^{(n)}(r)$ is not so easily bounded, and we need some new notations and other tools. For $P$ points $z_{1}, \ldots, \mathbf{z}_{P}$ in $I^{s}$ and a measurable subset $E$ of $I^{S}$ we need to estimate

$$
\left|\frac{A(E, \mathbf{Z})}{P}-\right| E|| \text {. }
$$

This is achieved by using Lemma 2.3 , which is due to Niederreiter and Wills [15]. If

$$
E=\left\{\left(x^{(0)}, \mathbf{x}^{\prime}\right) \in I^{s+1}: x^{(0)}<g\left(\mathbf{x}^{\prime}\right)\right\}
$$

(where $g$ is some positive function on $I^{s}$ ), we will use Lemma 2.3 in conjunction with Lemmas 2.4 and 2.5. After some preliminary results (Lemmas 2.6, 2.7, and 2.8) we obtain a bound for $\Delta_{N}^{(n)}(r)$ (Lemma 2.9). 
For $1 \leq i \leq N$ and $m \geq 0$ we deduce from (1.19)

$$
v_{i}^{(m)}<r \Leftrightarrow i \leq \sum_{j=1}^{N} \varphi_{r}\left(v_{j}^{(m)}\right) .
$$

If $\left(v, w, x^{(4)}\right) \in \mathbf{R}_{+}^{2} \times[0,1]$, we define

$$
\begin{aligned}
& g_{v, w}^{*}\left(x^{(4)}\right)=\min \left(\max \left(\frac{2 r^{2}-v^{2}-w^{2}}{2\left(\left(v^{2}+w^{2}\right)^{2}-4\left(2 x^{(4)}-1\right)^{2} v^{2} w^{2}\right)^{1 / 2}}+\frac{1}{2}, 0\right), 1\right), \\
& \text { if } v \neq w \text { or } v=w \neq 0 \text { and } x^{(4)} \neq 0,1 ; \text { otherwise } \\
& g_{v, v}^{*}(0)=g_{v, v}^{*}(1)=\varphi_{r}(v), \quad g_{0,0}^{*}\left(x^{(4)}\right)=1 .
\end{aligned}
$$

The function $g_{v, w}^{*}$ is continuous and satisfies

(2.15) $v \leq v^{\prime}, w \leq w^{\prime} \Rightarrow g_{v, w}^{*}\left(x^{(4)}\right) \geq g_{v^{\prime}, w^{\prime}}^{*}\left(x^{(4)}\right), \quad x^{(4)} \in[0,1]$,

$$
\left[v, w ; x^{(4)}, x^{(5)}\right]<r \Leftrightarrow x^{(5)}<g_{v, w}^{*}\left(x^{(4)}\right) .
$$

We set

$$
g_{i, j}^{(n)}=g_{v_{i}^{(n)}, v_{j}^{(n)}}^{*}, \quad 1 \leq i \leq N, 1 \leq j \leq N .
$$

For $1 \leq m \leq n$ let $\chi_{m}^{(n)}$ be the characteristic function of $\left[\frac{m-1}{n}, \frac{m}{n}\right)$. We introduce the sets

$$
\begin{aligned}
& E_{N}^{(n)}(r)=\left\{\mathbf{x} \in I^{6}: x^{(1)}<\frac{1}{N} \sum_{i=1}^{N} \sum_{m=0}^{n-1} \varphi_{r}\left(v_{i}^{(m)}\right) \chi_{m+1}^{(n)}\left(x^{(6)}\right)\right\}, \\
& F_{N}^{(n)}(r)=\left\{\mathbf{x} \in I^{6}: x^{(3)}<\sum_{i=1}^{N} \sum_{j=1}^{N} \sum_{m=0}^{n-1} \chi_{i, j}\left(x^{(1)}, x^{(2)}\right)\right. \\
& \left.\cdot \Delta t q\left(\left[v_{i}^{(m)}, v_{j}^{(m)} ; x^{(4)}\right]\right) \chi_{m+1}^{(n)}\left(x^{(6)}\right)\right\}, \\
& G_{N}^{(n)}(r)=\left\{\mathbf{x} \in I^{6}: x^{(5)}<\sum_{i=1}^{N} \sum_{j=1}^{N} \sum_{m=0}^{n-1} \chi_{i, j}\left(x^{(1)}, x^{(2)}\right)\right. \\
& \left.\cdot g_{i, j}^{(m)}\left(x^{(4)}\right) \chi_{m+1}^{(n)}\left(x^{(6)}\right)\right\},
\end{aligned}
$$

and

$$
\mathbf{X}_{n N}^{+}=\left\{\left(\mathbf{x}_{p}, \frac{p-1}{n N}\right): 1 \leq p \leq n N\right\} \subset I^{6}
$$


By (2.13) and (2.16),

$$
\begin{aligned}
\Delta_{N}^{(n)}(r)=n( & \frac{A\left(E_{N}^{(n)}(r) \cap F_{N}^{(n)}(r)^{c}, \mathbf{X}_{n N}^{+}\right)}{n N}-\left|E_{N}^{(n)}(r) \cap F_{N}^{(n)}(r)^{c}\right| \\
& \left.+\frac{A\left(F_{N}^{(n)}(r) \cap G_{N}^{(n)}(r), \mathbf{X}_{n N}^{+}\right)}{n N}-\left|F_{N}^{(n)}(r) \cap G_{N}^{(n)}(r)\right|\right) .
\end{aligned}
$$

We now state the Lemma of Niederreiter and Wills.

For a subset $E$ of $I^{s}$ and $\varepsilon>0$, we define

$$
\begin{gathered}
E_{\varepsilon}=\left\{\mathbf{z} \in I^{s}: \exists \mathbf{z}^{\prime} \in E,\left|\mathbf{z}-\mathbf{z}^{\prime}\right|<\varepsilon\right\}, \\
E_{-\varepsilon}=\left\{\mathbf{z} \in I^{s}: \forall \mathbf{z}^{\prime} \in I^{s} \backslash E,\left|\mathbf{z}-\mathbf{z}^{\prime}\right| \geq \varepsilon\right\} .
\end{gathered}
$$

Lemma 2.3. If $E$ is a measurable subset of $I^{s}$ such that

$$
\exists K>0 \forall \varepsilon>0 \max \left(\left|E_{\varepsilon} \backslash E\right|,\left|E \backslash E_{-\varepsilon}\right|\right) \leq K \varepsilon,
$$

then, for any $P$ points $\mathbf{z}_{1}, \ldots, \mathbf{z}_{P}$ in $I^{s}$,

$$
\left|\frac{A(E, \mathbf{Z})}{P}-\right| E|| \leq\left(4 K s^{1 / 2}+2 K+1\right) D_{P}(\mathbf{Z})^{1 / s} .
$$

If $E$ satisfies (2.23), we set

$$
K(E)=\inf \left\{K>0: \forall \varepsilon>0 \max \left(\left|E_{\varepsilon} \backslash E\right|,\left|E \backslash E_{-\varepsilon}\right|\right) \leq K \varepsilon\right\} .
$$

For a function $g$ on $I^{s}$ we denote by $V(g)$ its variation in the sense of Hardy and Krause (we refer to $[9,16]$ for the definition of this concept).

Lemma 2.4. If $g: I^{S} \rightarrow[0,1]$ is a function of bounded variation $V(g)$, and if

$$
E=\left\{\left(x^{(0)}, \mathbf{x}^{\prime}\right) \in I^{s+1}: x^{(0)}<g\left(\mathbf{x}^{\prime}\right)\right\}
$$

then

$$
K(E) \leq \frac{6^{s}-2^{s}+2}{2} s V(g)+1
$$

Proof. Given $\varepsilon>0$, let $N$ be the smallest integer such that $1 \leq N \varepsilon$. For $1 \leq i_{1} \leq N, \ldots, 1 \leq i_{s} \leq N$ let

$$
\begin{gathered}
I_{i_{1}, \ldots, i_{s}}=\left[\frac{i_{1}-1}{N}, \frac{i_{1}}{N}\right) \times \cdots \times\left[\frac{i_{s}-1}{N}, \frac{i_{s}}{N}\right), \\
m_{i_{1}, \ldots, i_{s}}=\inf \left\{g\left(\mathbf{x}^{\prime}\right): \mathbf{x}^{\prime} \in I_{i_{1}, \ldots, i_{s}}\right\}, \quad M_{i_{1}, \ldots, i_{s}}=\sup \left\{g\left(\mathbf{x}^{\prime}\right): \mathbf{x}^{\prime} \in I_{i_{1}, \ldots, i_{s}}\right\},
\end{gathered}
$$

and $\chi_{i_{1}, \ldots, i_{s}}$ be the characteristic function of $I_{i_{1}, \ldots, i_{s}}$. We define a new function $g^{*}$ by

$$
g^{*}\left(\mathbf{x}^{\prime}\right)=\sum_{i_{1}=1}^{N} \cdots \sum_{i_{s}=1}^{N} M_{i_{1}, \ldots, i_{s}} \chi_{i_{1}, \ldots, i_{s}}\left(\mathbf{x}^{\prime}\right), \quad \mathbf{x}^{\prime} \in I^{s}
$$


If

$$
\begin{gathered}
E^{*}=\left\{\left(x^{(0)}, \mathbf{x}^{\prime}\right) \in I^{s+1}: x^{(0)}<g^{*}\left(\mathbf{x}^{\prime}\right)\right\}, \\
F=\bigcup_{i_{1}=1}^{N} \cdots \bigcup_{i_{s}=1}^{N}\left[m_{i_{1}, \ldots, i_{s}}, M_{i_{1}, \ldots, i_{s}}\right] \times I_{i_{1}, \ldots, i_{s}},
\end{gathered}
$$

then

$$
E_{\varepsilon} \backslash E \subset\left(\left(E^{*}\right)_{\varepsilon} \backslash E^{*}\right) \cup F
$$

Let

$$
\begin{gathered}
g^{(\varepsilon)}\left(\mathbf{x}^{\prime}\right)=\max \left\{g^{*}\left(\mathbf{y}^{\prime}\right):\left|\mathbf{x}^{\prime}-\mathbf{y}^{\prime}\right|<\varepsilon\right\}, \quad \mathbf{x}^{\prime} \in I^{s}, \\
G^{(\varepsilon)}=\left\{\left(x^{(0)}, \mathbf{x}^{\prime}\right) \in I^{s+1}: g^{(\varepsilon)}\left(\mathbf{x}^{\prime}\right) \leq x^{(0)}<g^{(\varepsilon)}\left(\mathbf{x}^{\prime}\right)+\varepsilon\right\},
\end{gathered}
$$

and

$$
\begin{aligned}
H^{(\varepsilon)}=\bigcup_{i_{1}=1}^{N} \ldots \bigcup_{i_{s}=1}^{N} \bigcup_{j_{1}=i_{1}-1}^{i_{1}+1} \ldots \bigcup_{j_{s}=i_{s}-1}^{i_{s}+1}[ & \min \left(M_{i_{1}, \ldots, i_{s}}, M_{j_{1}, \ldots, j_{s}}\right), \\
& \left.\max \left(M_{i_{1}, \ldots, i_{s}}, M_{j_{1}, \ldots, j_{s}}\right)\right) \\
\times & \prod_{k=1}^{s} J_{i_{1}, \ldots, i_{s}, j_{1}, \ldots, j_{s}}^{(k)}
\end{aligned}
$$

where

$$
J_{i_{1}, \ldots, i_{s}, j_{1}, \ldots, j_{s}}^{(k)}= \begin{cases}{\left[\frac{i_{k}-1}{N}, \frac{i_{k}}{N}\right)} & \text { if } j_{k}=i_{k}, \\ {\left[\frac{i_{k}-1}{N}, \frac{i_{k}-1}{N}+\varepsilon\right)} & \text { if } j_{k}=i_{k}-1 \text { and } M_{i_{1}, \ldots, i_{s}}<M_{j_{1}, \ldots, j_{s}}, \\ \left(\frac{i_{k}}{N}-\varepsilon, \frac{i_{k}}{N}\right) & \text { if } j_{k}=i_{k}+1 \text { and } M_{i_{1}, \ldots, i_{s}}<M_{j_{1}, \ldots, j_{s}}, \\ \varnothing & \text { otherwise. }\end{cases}
$$

We have

$$
\left(E^{*}\right)_{\varepsilon} \backslash E^{*} \subset G^{(\varepsilon)} \cup H^{(\varepsilon)} .
$$

Now we use the following inequalities for estimating $\left|E_{\varepsilon} \backslash E\right|$ :

$$
|F| \leq \frac{s V(g)}{N}, \quad\left|G^{(\varepsilon)}\right| \leq \varepsilon, \quad\left|H^{(\varepsilon)}\right| \leq\left(3^{s}-1\right) s(\varepsilon N)^{s-1} V(g) \varepsilon .
$$

An estimate of $\left|E \backslash E_{-\varepsilon}\right|$ is similarly obtained.

Remark 2.1. If $s=1$, then $\left|H^{(\varepsilon)}\right|$ can be bounded by $V(g) \varepsilon$, consequently

$$
K(E) \leq 2 V(g)+1
$$

We specialize to obtain a more useful version of Lemma 2.4. Let $s \geq 2$ and $0 \leq r<s$ be integers. We choose $s-r$ integers $N_{r+1} \geq \cdots \geq N_{s}$. If $0<\varepsilon<1$, we set

$$
p(\varepsilon)= \begin{cases}\max \left\{p: r<p \leq s, N_{p} \geq \frac{1}{\varepsilon}\right\} & \text { if } N_{r+1} \geq \frac{1}{\varepsilon}, \\ r & \text { otherwise. }\end{cases}
$$


For $1 \leq i_{r+1} \leq N_{r+1}, \ldots, 1 \leq i_{s} \leq N_{s}$ and $r \leq p \leq s$ let

$$
\begin{gathered}
I_{i_{r+1}, \ldots, i_{p}}=\left[\frac{i_{r+1}-1}{N_{r+1}}, \frac{i_{r+1}}{N_{r+1}}\right) \times \cdots \times\left[\frac{i_{p}-1}{N_{p}}, \frac{i_{p}}{N_{p}}\right), \\
\mathbf{H}_{i_{r+1}, \ldots, i_{p}}^{(\varepsilon)}\left\{\left(j_{r+1}, \ldots, j_{p}\right): 1 \leq j_{r+1} \leq N_{r+1}, \ldots, 1 \leq j_{p} \leq N_{p},\right. \\
\left.\exists \mathbf{x}^{*} \in I_{i_{r+1}, \ldots, i_{p}} \exists \mathbf{y}^{*} \in I_{j_{r+1}, \ldots, j_{p}}\left|\mathbf{x}^{*}-\mathbf{y}^{*}\right|<\varepsilon\right\},
\end{gathered}
$$

and $\chi_{i_{r+1}, \ldots, i_{p}}$ be the characteristic function of $I_{i_{r+1}, \ldots, i_{p}}$. We use the Kronecker symbol $\delta$; if $h$ is a real-valued function, then $h^{+}$denotes its positive part.

Lemma 2.5. Let $E=\left\{\left(x^{(0)}, \mathbf{x}^{\prime}, \mathbf{x}^{\prime \prime}\right) \in I^{s+1}: x^{(0)}<g\left(\mathbf{x}^{\prime}, \mathbf{x}^{\prime \prime}\right)\right\}$, where

$$
\begin{gathered}
g\left(\mathbf{x}^{\prime}, \mathbf{x}^{\prime \prime}\right)=\sum_{i_{r+1}=1}^{N_{r+1}} \cdots \sum_{i_{s}=1}^{N_{s}} g_{i_{r+1}, \ldots, i_{s}}\left(\mathbf{x}^{\prime}\right) \chi_{i_{r+1}, \ldots, i_{s}}\left(\mathbf{x}^{\prime \prime}\right), \quad \mathbf{x}^{\prime} \in I^{r}, \mathbf{x}^{\prime \prime} \in I^{s-r}, \\
g_{i_{r+1}, \ldots, i_{s}}: I^{r} \rightarrow[0,1], \quad 1 \leq i_{r+1} \leq N_{r+1}, \ldots, 1 \leq i_{s} \leq N_{s} .
\end{gathered}
$$

For $1 \leq i_{r+1} \leq N_{r+1}, \ldots, 1 \leq i_{s} \leq N_{s}$ let

$$
\begin{aligned}
g_{i_{r+1}}^{(\varepsilon)}, \ldots, i_{s} & =\max \left\{g_{j_{r+1}, \ldots, j_{p(\varepsilon)}, i_{p(\ell)+1}, \ldots, i_{s}}:\left(j_{r+1}, \ldots, j_{p(\varepsilon)}\right) \in \mathbf{H}_{i_{r+1}, \ldots, i_{p(\varepsilon)}}^{(\varepsilon)}\right\} \\
g_{i_{r+1}, \ldots, i_{s}}^{(-\varepsilon)} & =\min \left\{g_{j_{r+1}, \ldots, j_{p(\varepsilon)}, i_{p(\varepsilon)+1}, \ldots, i_{s}}:\left(j_{r+1}, \ldots, j_{p(\varepsilon)}\right) \in \mathbf{H}_{i_{r+1}, \ldots, i_{p(\varepsilon)}}^{(\varepsilon)}\right\}
\end{aligned}
$$

If all the variations of $g_{i_{r+1}}^{(\varepsilon)}, \ldots, i_{s}$ and $g_{i_{r+1}, \ldots, i_{s}}^{(-\varepsilon)}, 1 \leq i_{r+1} \leq N_{r+1}, \ldots$, $1 \leq i_{s} \leq N_{s}, 0<\varepsilon<1$, are bounded by some $V$, then

$$
\begin{aligned}
\left|E_{\varepsilon} \backslash E\right| \leq & 3^{s-p(\varepsilon)}\left(\frac{6^{r}-2^{r}+2}{2} r V+1\right) \varepsilon \\
& +\sum_{i_{r+1}=1}^{N_{r+1}} \cdots \sum_{i_{s}=1}^{N_{s}} \sum_{j_{p(\varepsilon)+1}=i_{p(\varepsilon)+1}-1}^{i_{p(\varepsilon)+1}+1} \cdots \sum_{j_{s}=i_{s}-1}^{i_{s}+1} \\
& \cdot \prod_{k=r+1}^{p(\varepsilon)} \frac{1}{N_{k}} \prod_{k=p(\varepsilon)+1}^{s} \frac{\varepsilon}{\left(\varepsilon N_{k}\right)^{\delta_{i_{k},}, j_{k}}} \\
& \cdot \int_{I^{r}}\left(g_{i_{r+1}, \ldots, i_{p(\varepsilon)}(\varepsilon)}, j_{p(\varepsilon)+1}, \ldots, j_{s}\left(\mathbf{x}^{\prime}\right)-g_{i_{r+1}, \ldots, i_{s}}\left(\mathbf{x}^{\prime}\right)\right)^{+} d \mathbf{x}^{\prime}
\end{aligned}
$$


and

$$
\begin{aligned}
\left|E \backslash E_{-\varepsilon}\right| \leq & 3^{s-p(\varepsilon)}\left(\frac{6^{r}-2^{r}+2}{2} r V+1\right) \varepsilon \\
& +\sum_{i_{r+1}=1}^{N_{r+1}} \cdots \sum_{i_{s}=1}^{N_{s}} \sum_{j_{p(\varepsilon)+1}=i_{p(\varepsilon)+1}-1}^{i_{p(\varepsilon)+1}+1} \cdots \sum_{j_{s}=i_{s}-1}^{i_{s}+1} \\
& \cdot \prod_{k=r+1}^{p(\varepsilon)} \frac{1}{N_{k}} \prod_{k=p(\varepsilon)+1}^{s} \frac{\varepsilon}{\left(\varepsilon N_{k}\right)^{\delta_{i_{k}, j_{k}}}} \\
& \cdot \int_{I^{r}}\left(g_{i_{r+1}, \ldots, i_{s}}\left(\mathbf{x}^{\prime}\right)-g_{i_{r+1}, \ldots, i_{p(\varepsilon)}, j_{p(e)+1}, \ldots, j_{s}}^{(-\varepsilon)}\left(\mathbf{x}^{\prime}\right)\right)^{+} d \mathbf{x}^{\prime} .
\end{aligned}
$$

Proof. Let

$$
\begin{aligned}
F^{(\varepsilon)}= & \bigcup_{i_{r+1}=1}^{N_{r+1}} \ldots \bigcup_{i_{s}=1}^{N_{s}}\left(\left(F_{i_{r+1}, \ldots, i_{s}}^{(\varepsilon)}\right)_{\varepsilon} \backslash F_{i_{r+1}, \ldots, i_{s}}^{(\varepsilon)}\right) \\
& \times I_{i_{r+1}, \ldots, i_{p(\varepsilon)}} \times \prod_{k=p(\varepsilon)+1}^{s}\left(\frac{i_{k}-1}{N_{k}}-\varepsilon, \frac{i_{k}}{N_{k}}+\varepsilon\right), \\
G^{(\varepsilon)}= & \bigcup_{i_{r+1}=1}^{N_{r+1}} \ldots \bigcup_{i_{s}=1}^{N_{s}} \bigcup_{j_{p(\varepsilon)+1}=i_{p(\varepsilon)+1}-1}^{s} i_{j_{s}=i_{s}-1}^{i_{p(\varepsilon)+1}+1} G_{i_{r+1}, \ldots, i_{s}, j_{p(\varepsilon)+1}, \ldots, j_{s}}^{(\varepsilon)} J_{i_{s}+1}^{s} J_{i_{p(\varepsilon)+1}, \ldots, i_{s}, j_{p(\varepsilon)+1}, \ldots, j_{s},}^{(k)}
\end{aligned}
$$

where

$$
\begin{gathered}
F_{i_{r+1}, \ldots, i_{s}}^{(\varepsilon)}=\left\{\left(x^{(0)}, \mathbf{x}^{\prime}\right) \in I^{r+1}: x^{(0)}<g_{i_{r+1}, \ldots, i_{s}}^{(\varepsilon)}\left(\mathbf{x}^{\prime}\right)\right\}, \\
G_{i_{r+1}, \ldots, i_{s}, j_{p(e)+1}, \ldots, j_{s}}^{(\varepsilon)}=\left\{\left(x^{(0)}, \mathbf{x}^{\prime}\right) \in I^{r+1}: g_{i_{r+1}, \ldots, i_{s}}\left(\mathbf{x}^{\prime}\right) \leq x^{(0)}\right. \\
J_{i_{p(e)+1}, \ldots, i_{s}, j_{p(\varepsilon)+1}, \ldots, j_{s}}^{(k)}= \begin{cases}(\varepsilon) & \text { if } j_{k}=i_{k}, \\
{\left[\frac{i_{k+1}-1}{N_{k}}, \frac{i_{k}}{N_{k}}\right)} & \text { if } j_{k}=i_{k(\varepsilon)}, j_{p(\varepsilon)+1}, \ldots, j_{s} \\
\left.\left.N_{k}, \frac{i_{k}-1}{N_{k}}+\varepsilon\right)\right\}, \\
\left(\frac{i_{k}}{N_{k}}-\varepsilon, \frac{i_{k}}{N_{k}}\right) & \text { if } j_{k}=i_{k}+1 .\end{cases}
\end{gathered}
$$

We have

$$
E_{\varepsilon} \backslash E \subset F^{(\varepsilon)} \cup G^{(\varepsilon)}
$$

Inequality (2.27) then follows from Lemma 2.4 . Inequality $\left(2.27^{\prime}\right)$ is established similarly.

Remark 2.2. If $r=1$, then estimates $(2.27)$ and $\left(2.27^{\prime}\right)$ are improved by replacing $\left(6^{r}-2^{r}+2\right) r V / 2+1$ by $2 V+1$. 
The following lemmas provide us with bounds for $K\left(E_{N}^{(n)}(r)\right), K\left(F_{N}^{(n)}(r)\right)$, and $K\left(G_{N}^{(n)}(r)\right)$. We use the sets

$$
\begin{gathered}
\Phi_{N}^{(n)}(r)=\left\{\left(x^{(1)}, x^{(2)}, x^{(3)}, x^{(4)}, x^{(6)}\right) \in I^{5}:\right. \\
x^{(3)}<\sum_{i=1}^{N} \sum_{j=1}^{N} \sum_{m=0}^{n-1} \chi_{i, j}\left(x^{(1)}, x^{(2)}\right) \\
\left.\cdot \Delta t q\left(\left[v_{i}^{(m)}, v_{j}^{(m)} ; x^{(4)}\right]\right) \chi_{m+1}^{(n)}\left(x^{(6)}\right)\right\},
\end{gathered}
$$

$$
\mathbf{X}_{n N}^{\star}=\left\{\left(x_{p}^{(1)}, x_{p}^{(2)}, x_{p}^{(3)}, x_{p}^{(4)}, \frac{p-1}{n N}\right): 1 \leq p \leq n N\right\} \subset I^{5}
$$

For the sake of simplicity we now restrict ourselves to $n \leq N$, which is in accordance with the hypothesis of Theorem 2.1 below.

Lemma 2.6. The constant (2.25) associated with the set (2.17) can be estimated by

$$
\begin{aligned}
K\left(E_{N}^{(n)}(r)\right) \leq & 3+4 Q t^{(n-1)}+4\left(\left(4 \cdot 5^{1 / 2}+2\right)\left(90+9 Q t^{(n-1)}\right)+1\right) \\
& \cdot(n-1) D_{(n-1) N}\left(\mathbf{X}_{(n-1) N}^{\star}\right)^{1 / 5} .
\end{aligned}
$$

Proof. Inequality (2.27) leads to

where

$$
\left|\left(E_{N}^{(n)}(r)\right)_{\varepsilon} \backslash E_{N}^{(n)}(r)\right| \leq \begin{cases}3 \varepsilon+\Sigma \varepsilon & \text { if } \varepsilon<1 / n \\ \varepsilon+4 \Sigma \varepsilon & \text { if } \varepsilon \geq 1 / n\end{cases}
$$

By using

$$
\Sigma=\frac{1}{N} \sum_{m=1}^{n-1}\left|\sum_{i=1}^{N}\left(\varphi_{r}\left(v_{i}^{(m)}\right)-\varphi_{r}\left(v_{i}^{(m-1)}\right)\right)\right|
$$

$$
\Sigma \leq \frac{1}{N} A\left(\Phi_{N}^{(n-1)}(r), \mathbf{X}_{(n-1) N}^{\star}\right)
$$

together with inequality (2.24), we obtain an estimate for $\left|\left(E_{N}^{(n)}(r)\right)_{\varepsilon} \backslash E_{N}^{(n)}(r)\right|$. The same estimate is valid for $\left|E_{N}^{(n)}(r) \backslash\left(E_{N}^{(n)}(r)\right)_{-\varepsilon}\right|$. Then, since $K\left(\Phi_{N}^{(n-1)}(r)\right)=$ $K\left(F_{N}^{(n-1)}(r)\right)$, the desired result follows from Lemma 2.7 .

Lemma 2.7. The constant (2.25) associated with the set (2.18) satisfies the inequality

$$
K\left(F_{N}^{(n)}(r)\right) \leq 90+9 Q t^{(n-1)} .
$$

Proof. Using estimate (2.27) together with inequality (1.12), we obtain

$$
\left|\left(F_{N}^{(n)}(r)\right)_{\varepsilon} \backslash F_{N}^{(n)}(r)\right| \leq \begin{cases}\left(90+9 Q t^{(n-1)}\right) \varepsilon & \text { if } \varepsilon<\frac{1}{N} \\ \left(17+2 Q t^{(n-1)}\right) \varepsilon & \text { if } \frac{1}{N} \leq \varepsilon<\frac{1}{n} \\ \left(4+Q t^{(n-1)}\right) \varepsilon & \text { if } \frac{1}{n} \leq \varepsilon\end{cases}
$$

The same estimates are valid for $\left|F_{N}^{(n)}(r) \backslash\left(F_{N}^{(n)}(r)\right)_{-\varepsilon}\right|$. 
Lemma 2.8. The constant (2.25) associated with the set (2.19) can be estimated by

$$
\begin{aligned}
K\left(G_{N}^{(n)}(r)\right) \leq & 99+18 Q t^{(n-1)}+18\left(\left(4 \cdot 5^{1 / 2}+2\right)\left(90+9 Q t^{(n-1)}\right)+1\right) \\
& \cdot(n-1) D_{(n-1) N}\left(\mathbf{X}_{n-1) N}^{\star}\right)^{1 / 5} .
\end{aligned}
$$

Proof. Inequality (2.27) leads to

$$
\left|\left(G_{N}^{(n)}(r)\right)_{\varepsilon} \backslash G_{N}^{(n)}(r)\right| \leq \begin{cases}(99+9 \Theta) \varepsilon & \text { if } \varepsilon<\frac{1}{N} \\ (21+\Theta) \varepsilon & \text { if } \frac{1}{N} \leq \varepsilon<\frac{1}{n} \\ (7+4 \Theta) \varepsilon & \text { if } \frac{1}{n} \leq \varepsilon\end{cases}
$$

where

$$
\Theta=\frac{1}{N^{2}} \sum_{i=1}^{N} \sum_{j=1}^{N} \sum_{m=1}^{n-1} \int_{0}^{1}\left|g_{i, j}^{(m)}\left(x^{(4)}\right)-g_{i, j}^{(m-1)}\left(x^{(4)}\right)\right| d x^{(4)}
$$

Because of (2.16) we have

$$
\begin{aligned}
& \int_{0}^{1}\left|g_{i, j}^{(m)}\left(x^{(4)}\right)-g_{i, j}^{(m-1)}\left(x^{(4)}\right)\right| d x^{(4)} \\
& \quad=\int_{I^{2}} \mid \varphi_{r}\left(\left[v_{i}^{(m)}, v_{j}^{(m)} ; x^{(4)}, x^{(5)}\right]\right) \\
& \quad \quad-\varphi_{r}\left(\left[v_{i}^{(m-1)}, v_{j}^{(m-1)} ; x^{(4)}, x^{(5)}\right]\right) \mid d x^{(4)} d x^{(5)} .
\end{aligned}
$$

Thus, using (2.1),

$$
\Theta \leq \frac{2}{N} A\left(\Phi_{N}^{(n-1)}(r), \mathbf{X}_{(n-1) N}^{\star}\right),
$$

and an estimate of $\left|\left(G_{N}^{(n)}(r)\right)_{\varepsilon} \backslash G_{N}^{(n)}(r)\right|$ follows by Lemma 2.3. The same estimate is valid for $\left|G_{N}^{(n)}(r) \backslash\left(G_{N}^{(n)}(r)\right)_{-\varepsilon}\right|$. Then, since

$$
K\left(\Phi_{N}^{(n-1)}(r)\right)=K\left(F_{N}^{(n-1)}(r)\right),
$$

the desired conclusion is a consequence of Lemma 2.7.

Using (2.21) together with the results of Lemmas 2.3, 2.6, 2.7, and 2.8, we obtain the following bound for $\Delta_{N}^{(n)}(r)$.

Lemma 2.9. The error term (2.7) satisfies the inequality

$$
\begin{aligned}
\left|\Delta_{N}^{(n)}(r)\right| \leq & ( \\
+ & \left.\cdot 6^{1 / 2}+2\right)\left(282+40 Q t^{(n-1)}\right. \\
& +22\left(\left(4 \cdot 5^{1 / 2}+2\right)\left(90+9 Q t^{(n-1)}\right)+1\right) \\
& \left.\left.\cdot(n-1) D_{(n-1) N}\left(\mathbf{X}_{(n-1) N}^{\star}\right)^{1 / 5}\right)+2\right) n D_{n N}\left(\mathbf{X}_{n N}^{+}\right)^{1 / 6} .
\end{aligned}
$$

We are now able to prove the convergence of the QMC method. Consider the Boltzmann equation in $\mathbf{R}_{+} \times(0, T)$. We divide the time interval $(0, T)$ into $P$ subintervals of length $\Delta t$. 
Theorem 2.1. Let $\mathbf{S}$ be a subset of $\mathbf{N}^{2}$, and let the following conditions hold:

$$
\begin{gathered}
\lim _{N \rightarrow \infty} D_{N}^{*}\left(V^{(0)}, v^{2} f_{0}\right)=0, \\
\lim _{(N, P) \in \mathbf{S}, N \& P \rightarrow \infty} \max _{1 \leq n \leq P} n D_{n N}\left(\mathbf{X}_{n N}^{+}\right)^{1 / 6}=0 .
\end{gathered}
$$

Then

$$
\lim _{(N, P) \in \mathbf{S}, N \& P \rightarrow \infty} \max _{1 \leq n \leq P} D_{N}^{*}\left(V^{(n)}, v^{2} f_{n}\right)=0 .
$$

Proof. If we use equation (2.9) and estimates (2.11) and (2.12), we obtain the inequality

$$
\begin{aligned}
D_{N}^{*}\left(V^{(n)}, v^{2} f_{n}\right) \leq & e^{11 Q t^{(n)}} D_{N}^{*}\left(V^{(0)}, v^{2} f_{0}\right) \\
& +\Delta t \int_{\left(0, t^{(n)}\right) \times \mathbf{R}_{+}} e^{11 Q\left(t^{(n)}-t\right)} v^{2}\left|\frac{\partial^{2} f}{\partial t^{2}}(v, t)\right| d v d t \\
& +e^{11 Q t^{(n-1)}} \max _{1 \leq m \leq n} \sup _{r>0}\left|\Delta_{N}^{(m)}(r)\right|, \quad n \geq 1 .
\end{aligned}
$$

Using (2.33), we have the result.

Remark 2.3. (i) If estimate (2.35) is used in conjunction with Lemma 2.9, we obtain an effective error bound for the QMC method.

(ii) The *-discrepancy $D_{n N}^{*}\left(\mathbf{X}_{n N}^{+}\right)$can be shown [17] to satisfy the inequality

$$
D_{n N}^{*}\left(\mathbf{X}_{n N}^{+}\right) \leq \frac{1}{n N}\left(\max _{1 \leq P \leq n N} P D_{P}^{*}(\mathbf{X})+1\right) \text {. }
$$

(iii) Halton was the first to show [6] that for any dimension $s \geq 1$ there exists a sequence $\mathbf{H}$ of points in $I^{s}$ with

$$
D_{P}(\mathbf{H})=\frac{O\left((\log P)^{s}\right)}{P}, \quad P \geq 2,
$$

and it is a widely-held belief that the order of magnitude in (2.37) is best possible.

\section{Computational Results}

Computer implementation of the QMC method requires some sequence $\mathbf{X}$ which satisfies conditions (1.18) and $\left(1.18^{\prime}\right)$. The construction of $\mathbf{X}$ is based on the theory of $(0, s)$-sequences, which was developed by Niederreiter [17]. We propose here an algorithm for generating $(0, s)$-sequences, which is suited to vector computers. Then a convenient sequence $\mathbf{X}$ is constructed by a symmetrization of a $(0,5)$-sequence. Its $*$-discrepancy is estimated by means of the $*$-discrepancy of the $(0,5)$-sequence which is used.

Let $s \geq 1$ and $b \geq 2$ be integers. An elementary interval in base $b$ is an interval of the form

$$
J=\prod_{i=1}^{s}\left[\frac{a^{(i)}}{b^{d_{i}}}, \frac{a^{(i)}+1}{b^{d_{i}}}\right),
$$

with integers $d_{i} \geq 0$ and integers $0 \leq a^{(i)}<b^{d_{i}}$ for $1 \leq i \leq s$. 
Let $m$ be an integer. A point set $\left\{\mathbf{x}_{1}, \ldots, \mathbf{x}_{b^{m}}\right\} \subset I^{s}$ is a $(0, m, s)$-net in base $b$ if $A(J, \mathbf{X})=1$ for every elementary interval $J$ in base $b$ with $|J|=b^{-m}$.

A sequence $\mathbf{x}_{1}, \mathbf{x}_{2}, \ldots$ of points in $I^{s}$ is a $(0, s)$-sequence in base $b$ if for all integers $k \geq 0$ and $m>0$ the point set $\left\{\mathbf{x}_{p}: k b^{m}<p \leq(k+1) b^{m}\right\}$ is a $(0, m, s)$-net in base $b$.

The *-discrepancy of the first $P$ terms of a $(0, s)$-sequence in base $b \geq 3$ can be shown [17] to satisfy

$$
\begin{aligned}
D_{P}^{*}(\mathbf{X}) \leq \frac{1}{P}( & \frac{b-1}{2} \sum_{i=1}^{s}\left(\begin{array}{c}
s-1 \\
i-1
\end{array}\right)\left(\begin{array}{c}
k+1 \\
i
\end{array}\right)\left\lfloor\frac{b}{2}\right\rfloor^{i-1} \\
& \left.+\frac{1}{2} \sum_{i=0}^{s-1}\left(\begin{array}{c}
s-1 \\
i
\end{array}\right)\left(\left(\begin{array}{c}
k+1 \\
i
\end{array}\right)+\left(\begin{array}{c}
k \\
i
\end{array}\right)\right)\left\lfloor\frac{b}{2}\right\rfloor^{i}\right),
\end{aligned}
$$

where $P \geq 1$ and the integer $k$ is determined by $b^{k} \leq P<b^{k+1}$.

Faure was the first to show [5] that for any prime $b$ and any $s \leq b$ there exists a $(0, s)$-sequence in base $b$. Let $F_{b}$ be the set $\{0, \ldots, b-1\}$ endowed with the field structure induced by the arithmetic of integers with reduction modulo $b$. We can choose $s$ distinct elements $b_{1}, \ldots, b_{s}$ of $F_{b}$. For $p \geq 1$ let

$$
p-1=\sum_{j=0}^{\infty} a_{j}(p) b^{j}, \quad a_{j}(p) \in F_{b},
$$

be the representation of $p-1$ in base $b$. We define $\mathbf{y}_{p}=\left(y_{p}^{(1)}, \ldots, y_{p}^{(s)}\right)$ by

$$
y_{p}^{(i)}=\sum_{j=1}^{\infty} y_{p, j}^{(i)} b^{-j}, \quad 1 \leq i \leq s,
$$

with

$$
y_{p, j}^{(i)}=\sum_{k=j-1}^{\infty}\left(\begin{array}{c}
k \\
j-1
\end{array}\right) b_{i}^{k-j+1} a_{k}(p) \in F_{b}, \quad 1 \leq i \leq s, j \geq 1 .
$$

We propose an algorithm for computer generation of $\mathbf{Y}=\left\{\mathbf{y}_{p}: p \geq 1\right\}$ that generalizes an algorithm used for generating the Halton sequence [12]. Let $\lambda \geq 1$ be an integer and $\nu=b^{\lambda}$. The point sets

$$
\mathbf{Y}^{(n)}=\left\{\mathbf{y}_{p}: n \nu<p \leq(n+1) \nu\right\}, \quad n \geq 0,
$$

will be successively generated:

(i) Computation of $\mathbf{Y}^{(0)}$. Let $1 \leq m<b$ and $1 \leq l<\lambda$ be integers. For any integer $p, m b^{l}<p \leq(m+1) b^{l}$, the representation of $p-1$ in base $b$ can be deduced from the representation of $p-m b^{l}-1$. Hence, for $1 \leq i \leq s$ 
the digits of $y_{p}^{(i)}$ in base $b$ are related to the digits of $y_{p-m b^{l}}^{(i)}$ by

$$
y_{p, j}^{(i)}=y_{p-m b^{l}, j}^{(i)}+\left(\begin{array}{c}
l \\
j-1
\end{array}\right) b_{i}^{l-j+1} m, \quad 1 \leq j \leq l+1 .
$$

Relation (3.5) leads to recurrences regarding arrays of growing length:

$$
\left(y_{b^{l}+1, j}^{(i)}, \ldots, y_{b^{l+1}, j}^{(i)}\right), \quad 1 \leq i \leq s, 1 \leq j \leq l+1, l \leq l<\lambda .
$$

(ii) Computation of $\mathbf{Y}^{(n)}, n \geq 1$. Let

$$
n=\sum_{j=0}^{n^{\prime}} a_{j}(n+1) b^{j}
$$

be the representation of $n$ in base $b$. For any integer $p, n \nu<p \leq(n+1) \nu$, the representation of $p-1$ in base $b$ is related to the representation of $p-n \nu-1$. It follows that, for $1 \leq i \leq s$ and $1 \leq j \leq \lambda+n^{\prime}+1$,

$$
y_{p, j}^{(i)}=y_{p-n \nu, j}^{(i)}+\sum_{k=\max (j-1, \lambda)}^{\lambda+n^{\prime}}\left(\begin{array}{c}
k \\
j-1
\end{array}\right) b_{i}^{k-j+1} a_{k-\lambda}(n+1) \text {. }
$$

If the digits $y_{p, j}^{(i)}, 1 \leq i \leq s, 1 \leq p \leq \nu, 1 \leq j \leq \lambda$, are stored, (3.6) is used for computing the following integer arrays:

$$
\left(y_{n \nu+1, j}^{(i)}, \ldots, y_{(n+1) \nu, j}^{(i)}\right), \quad 1 \leq i \leq s, 1 \leq j \leq \lambda+n^{\prime}+1 .
$$

To use the QMC method, we consider the case $s=5$ and $b=5$. We select the number $N$ of simulated molecules in the form $N=2 b^{\lambda}$, where $\lambda$ is some integer, and we set $\nu=b^{\lambda}$. If $\mathbf{Y}$ is a $(0,5)$-sequence in base $b$, then another sequence $\mathbf{X}$ of points in $I^{5}$ is constructed by the following procedure. For $m \geq 0$, define

$$
\begin{array}{r}
\mathbf{x}_{p}=\left(\frac{1}{2}\left(y_{p^{*}}^{(1)}+\frac{\left\lfloor\nu y_{p^{*}}^{(1)}\right\rfloor+1}{\nu}\right), \frac{1}{2}\left(y_{p^{*}}^{(2)}+\frac{\left\lfloor\nu y_{p^{*}}^{(2)}\right\rfloor}{\nu}\right), y_{p^{*}}^{(3)}, y_{p^{*}}^{(4)},\right. \\
\left.\frac{1}{2}\left(y_{p^{*}}^{(5)}+\frac{\left\lfloor\nu y_{p^{*}}^{(5)}\right\rfloor+1}{\nu}\right)\right)
\end{array}
$$

(where $\left.p^{*}=p-m \nu\right)$, if $2 m \nu<p \leq(2 m+1) \nu$;

$$
\begin{array}{r}
\mathbf{x}_{p}=\left(\frac{1}{2}\left(y_{p^{*}}^{(2)}+\frac{\left\lfloor\nu y_{p^{*}}^{(2)}\right\rfloor}{\nu}\right), \frac{1}{2}\left(y_{p^{*}}^{(1)}+\frac{\left\lfloor\nu y_{p^{*}}^{(1)}\right\rfloor+1}{\nu}\right), y_{p^{*}}^{(3)}, y_{p^{*}}^{(4)},\right. \\
\left.1-\frac{1}{2}\left(y_{p^{*}}^{(5)}+\frac{\left\lfloor\nu y_{p^{*}}^{(5)}\right\rfloor+1}{\nu}\right)\right)
\end{array}
$$

$\left(\right.$ where $\left.p^{*}=p-(m+1) \nu\right)$, if $(2 m+1) \nu<p \leq 2(m+1) \nu$. 
Conditions (1.18) and (1.18') are obviously satisfied by $\mathbf{X}$. Observe that no point of $\mathbf{X}$ lies in $\left[\frac{i-1}{N}, \frac{i}{N}\right)^{2} \times I^{3}, 1 \leq i \leq N$. Consequently,

a molecule cannot collide with itself,

$$
D_{P}^{*}(\mathbf{X}) \geq \frac{1}{N^{2}}, \quad P \geq 1 .
$$

In fact, the $*$-discrepancy of $\mathbf{X}$ can be estimated by means of the $*$-discrepancy of $\mathbf{Y}$.

Lemma 3.1. The $*$-discrepancy $D_{n N}^{*}(\mathbf{X})$ of the first $n N$ terms of $\mathbf{X}$ satisfies

$$
D_{n N}^{*}(\mathbf{X}) \leq \frac{3}{2} D_{n \nu}^{*}(\mathbf{Y})+\frac{2}{N^{2}}, \quad n \geq 1 .
$$

Proof. Let $n=\sum_{j=0}^{n^{\prime}} a_{j} b^{j}$ be the representation of $n$ in base $b$. We split up the point set $\mathbf{X}_{n N}=\left\{\mathbf{x}_{p}: 1 \leq p \leq n N\right\}$ into the point sets

$$
\mathbf{X}_{j, k}=\left\{\mathbf{x}_{p}: n_{j, k} N<p \leq\left(n_{j, k}+1\right) N\right\}, \quad 0 \leq j \leq n^{\prime}, 0 \leq k<a_{j} b^{j},
$$

where

$$
n_{j, k}=k+\sum_{i=j+1}^{n^{\prime}} a_{i} b^{i} .
$$

Similarly, the point set $\mathbf{Y}_{n \nu}=\left\{\mathbf{y}_{p}: 1 \leq p \leq n \nu\right\}$ can be split up into the point sets

$$
\mathbf{Y}_{j, k}=\left\{\mathbf{y}_{p}: n_{j, k} \nu<p \leq\left(n_{j, k}+1\right) \nu\right\}, \quad 0 \leq j \leq n^{\prime}, 0 \leq k<a_{j} b^{j} .
$$

Let $J=\prod_{i=1}^{5}\left[0, u^{(i)}\right)$ be a subinterval of $I^{5}$. For $h=1,2$ consider the intervals

$$
J_{h}=\prod_{i=1}^{5} J_{h}^{(i)}
$$

with

$$
\begin{aligned}
& J_{h}^{(1)}=\left[0, \max \left(\frac{\left\lfloor\nu u^{(h)}\right\rfloor}{\nu}, 2 u^{(h)}-\frac{\left\lfloor\nu u^{(h)}\right\rfloor+1}{\nu}\right)\right), \\
& J_{h}^{(2)}=\left[0, \min \left(\frac{\left\lfloor\nu u^{(g)}\right\rfloor+1}{\nu}, 2 u^{(g)}-\frac{\left\lfloor\nu u^{(g)}\right\rfloor}{\nu}\right)\right) \\
& J_{h}^{(i)}=\left[0, u^{(i)}\right), \quad i=3,4,
\end{aligned}
$$




$$
\begin{aligned}
& J_{1}^{(5)}=\left[0, \max \left(\frac{\left\lfloor\nu u^{(5)}\right\rfloor}{\nu}, 2 u^{(5)}-\frac{\left\lfloor\nu u^{(5)}\right\rfloor+1}{\nu}\right)\right) \text {, }
\end{aligned}
$$

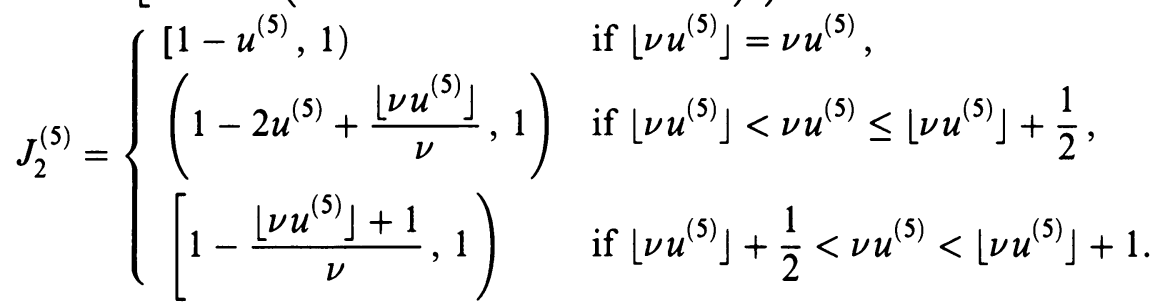

Then

$$
A\left(J, \mathbf{X}_{j, k}\right)=A\left(J_{1}, \mathbf{Y}_{j, k}\right)+A\left(J_{2}, \mathbf{Y}_{j, k}\right), \quad 0 \leq j \leq n^{\prime}, 0 \leq k<a_{j} b^{j}
$$

and

$$
|2| J|-| J_{1}|-| J_{2}|| \leq 1 / \nu^{2}
$$

Finally, we observe that

$$
\left|A\left(J_{h}, \mathbf{Y}_{n \nu}\right)-n \nu\right| J_{h}|| \leq h n \nu D_{n \nu}^{*}(\mathbf{Y}), \quad h=1,2
$$

and the required result now follows.

Next we consider the question of approximating the initial velocity distribution. It has been shown [9] that $D_{N}^{*}(X) \geq 1 / 2 N$ for any $N$ numbers $x_{1}, \ldots, x_{N}$ in $I$, and that the lower bound is attained for the point set

$$
U=\{(2 i-1) / 2 N: 1 \leq i \leq N\}
$$

The function

$$
F_{0}: v \rightarrow \int_{0}^{v} u^{2} f_{0}(u) d u
$$

is a strictly increasing function from $\mathbf{R}_{+}$onto $I$, and so the inverse function $F_{0}^{-1}=G_{0}$ exists. We define the initial velocities by

$$
v_{i}^{(0)}=G_{0}((2 i-1) / 2 N), \quad 1 \leq i \leq N .
$$

It is easily seen that

$$
D_{N}^{*}\left(V^{(0)}, v^{2} f_{0}\right)=D_{N}^{*}(U)=1 / 2 N
$$

We wish to assess the accuracy of the QMC method through computation of effective errors in an example where an exact solution is known. Krook and $\mathrm{Wu}$ [8] have discovered an exact solution of the Boltzmann equation, in the simplified case to which the present analysis is restricted. The solution was obtained by assuming

$$
\begin{gathered}
f_{0}(v)=\frac{20 \pi}{9}\left(\frac{5}{6 \pi}\right)^{3 / 2} v^{2} \exp \left(-\frac{5}{6} v^{2}\right), \quad v \in \mathbf{R}_{+}, \\
q(g)=6, \quad g \in \mathbf{R}_{+} .
\end{gathered}
$$


Then

$$
\begin{aligned}
f(v, t)= & \frac{4}{(2 \pi)^{1 / 2} H(t)^{5 / 2}}\left(5 H(t)-3+\frac{1-H(t)}{H(t)} v^{2}\right) \\
& \cdot \exp \left(-\frac{v^{2}}{2 H(t)}\right), \quad v, t \in \mathbf{R}_{+},
\end{aligned}
$$

where $H(t)=1-\frac{2}{5} \exp (-t)$.

The system is near equilibrium at $T=1.5$, so that computations were carried out up to $T=1.5$. We examine the effects of the number $N$ of simulated molecules and the number $P$ of time steps upon the accuracy of the approximation. These effects are examined by computing the errors $D_{N}^{*}\left(V^{(P)}, v^{2} f_{P}\right)$. For reasons of comparison we also applied the KMC scheme. A KMC calculation is based on pseudorandom numbers. Sequences of pseudorandom numbers are generated here by the routine G05CAF of the NAG [13]. The routine uses a multiplicative congruential method and the period of the generator is $2^{57}$. All computations were carried out on a CYBER 180-990 computer. The values of $D_{N}^{*}\left(V^{(P)}, v^{2} f_{P}\right)$ are listed in Table 1 for the QMC method and in Table 2 for the KMC scheme. The corresponding timing results are given in Table 3 for the QMC method and in Table 4 for the KMC scheme. From the results presented in Table 1, the following observations can be made: (i) for all $P$, the error of the QMC method regularly decreases if the number $N$ of simulated molecules increases, (ii) for each $N$, the error is oscillating when the stepsize decreases. It is also true that, in general, high accuracy is obtained for large $P$. On the other hand, from Table 2 we see that (i) for all $P$ the error of the KMC scheme irregularly decreases as $N$ increases, (ii) for each $N$ the error is oscillating as $P$ increases. Unlike in Table 1 , very poor accuracy is obtained for large $P$. For all $P$ it is clear that the QMC solution converges to the exact solution faster, for an equal number of simulated molecules, than the KMC solution. Moreover, it may be concluded from Tables 3 and 4 that the QMC method shows a substantial gain in efficiency (higher accuracy at the same costs) when compared

\begin{tabular}{|c|c|c|c|c|c|c|c|}
\hline \\
\hline$N$ & 10 & 50 & 250 & 1250 & 6250 & 31250 & 156250 \\
\hline 6 & $80 E-1$ & $9.05 E-2$ & $3.09 E-2$ & $1.05 E-2$ & $4.36 \mathrm{E}-3$ & $2.19 E-3$ & $1.34 \mathrm{E}-3$ \\
\hline 32 & $2.32 \mathrm{E}-1$ & $6.12 E-2$ & $2.46 \mathrm{E}-2$ & $1.03 E-2$ & $5.29 E-3$ & $1.85 \mathrm{E}-3$ & $1.13 E-3$ \\
\hline 4 & $1.98 \mathrm{E}-1$ & $5.39 E-2$ & $2.71 \mathrm{E}-2$ & $1.29 \mathrm{E}-2$ & $4.86 \mathrm{E}-3$ & $1.60 E-3$ & $6.24 E-4$ \\
\hline 28 & $2.65 \mathrm{E}-1$ & $1.03 E-1$ & $3.61 \mathrm{E}-2$ & $1.13 \mathrm{E}-2$ & $4.21 \mathrm{E}-3$ & $1.68 \mathrm{E}-3$ & $8.36 E-4$ \\
\hline 56 & $1.94 \mathrm{E}-1$ & $8.36 \mathrm{E}-2$ & $2.73 E-2$ & $1.33 \mathrm{E}-2$ & $3.98 \mathrm{E}-3$ & $2.15 \mathrm{E}-3$ & $6.23 E-4$ \\
\hline 12 & $1.80 \mathrm{E}-1$ & $7.05 E-2$ & $3.31 \mathrm{E}-2$ & $1.13 E-2$ & $3.44 \mathrm{E}-3$ & $1.45 \mathrm{E}-3$ & $5.18 \mathrm{E}-4$ \\
\hline 1024 & $1.81 \mathrm{E}-1$ & $6.41 E-2$ & $2.19 \mathrm{E}-2$ & $1.36 \mathrm{E}-2$ & $4.21 \mathrm{E}-3$ & $1.30 \mathrm{E}-3$ & $4.48 \mathrm{E}-4$ \\
\hline
\end{tabular}
with the KMC scheme. 
TABLE 2

The $D_{N}^{*}\left(V^{(P)}, v^{2} f_{P}\right)$ values for the KMC scheme

$\begin{array}{cccccccc}N & 10 & 50 & 250 & 1250 & 6250 & 31250 & 156250 \\ P & & & & & & & \\ 16 & 1.85 \mathrm{E}-1 & 6.76 \mathrm{E}-2 & 4.79 \mathrm{E}-2 & 3.39 \mathrm{E}-2 & 7.73 \mathrm{E}-3 & 3.12 \mathrm{E}-3 & 1.35 \mathrm{E}-3 \\ 32 & 2.33 \mathrm{E}-1 & 9.03 \mathrm{E}-2 & 6.64 \mathrm{E}-2 & 2.29 \mathrm{E}-2 & 8.70 \mathrm{E}-3 & 4.19 \mathrm{E}-3 & 2.15 \mathrm{E}-3 \\ 64 & 1.87 \mathrm{E}-1 & 1.12 \mathrm{E}-1 & 4.81 \mathrm{E}-2 & 2.69 \mathrm{E}-2 & 7.44 \mathrm{E}-3 & 6.35 \mathrm{E}-3 & 1.41 \mathrm{E}-3 \\ 128 & 2.09 \mathrm{E}-1 & 6.15 \mathrm{E}-2 & 3.67 \mathrm{E}-2 & 2.56 \mathrm{E}-2 & 8.81 \mathrm{E}-3 & 4.77 \mathrm{E}-3 & 1.51 \mathrm{E}-3 \\ 256 & 2.06 \mathrm{E}-1 & 6.20 \mathrm{E}-2 & 3.70 \mathrm{E}-2 & 2.30 \mathrm{E}-2 & 8.44 \mathrm{E}-3 & 2.53 \mathrm{E}-3 & 1.31 \mathrm{E}-3 \\ 512 & 2.62 \mathrm{E}-1 & 9.93 \mathrm{E}-2 & 7.76 \mathrm{E}-2 & 2.00 \mathrm{E}-2 & 9.12 \mathrm{E}-3 & 2.02 \mathrm{E}-3 & 1.95 \mathrm{E}-3 \\ 1024 & 1.73 \mathrm{E}-1 & 1.36 \mathrm{E}-1 & 6.40 \mathrm{E}-2 & 2.74 \mathrm{E}-2 & 1.05 \mathrm{E}-2 & 5.91 \mathrm{E}-3 & 1.57 \mathrm{E}-3\end{array}$

\section{TABLE 3}

$C P U$ times in seconds for the QMC method

$\begin{array}{llllllll}\quad N & 10 & 50 & 250 & 1250 & 6250 & 31250 & 156250 \\ P & & & & & & & \\ 16 & .01 & .04 & .22 & 1.21 & 6.64 & 35.85 & 193.84 \\ 32 & .01 & .06 & .30 & 1.68 & 9.53 & 52.81 & 293.65 \\ 64 & .02 & .08 & .44 & 2.60 & 15.30 & 87.00 & 492.64 \\ 128 & .03 & .13 & .72 & 4.44 & 26.86 & 155.41 & 892.96 \\ 256 & .07 & .24 & 1.31 & 8.16 & 50.11 & 294.28 & 1697.21 \\ 512 & .13 & .45 & 2.46 & 15.57 & 96.48 & 571.24 & 3311.92 \\ 1024 & .28 & .90 & 4.82 & 30.56 & 189.29 & 1128.63 & 6623.83\end{array}$

TABLE 4

$C P U$ times in seconds for the KMC scheme

$\begin{array}{llllllll}N & 10 & 50 & 250 & 1250 & 6250 & 31250 & 156250 \\ P & & & & & & & \\ 16 & .01 & .04 & .19 & .93 & 4.71 & 23.49 & 117.97 \\ 32 & .01 & .05 & .23 & 1.13 & 5.70 & 28.60 & 146.45 \\ 64 & .02 & .06 & .31 & 1.54 & 7.84 & 39.34 & 204.99 \\ 128 & .02 & .10 & .47 & 2.35 & 11.79 & 60.75 & 315.77 \\ 256 & .04 & .17 & .81 & 4.07 & 19.96 & 123.82 & 537.47 \\ 512 & .07 & .30 & 1.45 & 7.16 & 36.51 & 185.83 & 989.33 \\ 1024 & .13 & .57 & 2.79 & 13.65 & 69.09 & 352.59 & 1823.71\end{array}$

\section{BIBLIOGRAPHY}

1. H. Babovsky, F. Gropengiesser, H. Neunzert, J. Struckmeier, and B. Wiesen, Low discrepancy methods for the Boltzmann equation, 16th Internat. Sympos. on Rarefied Gas Dynamics, Pasadena, Ca., July 1988.

2. G. A. Bird, Molecular gas dynamics, Clarendon Press, Oxford, 1976.

3. _ Monte Carlo simulation in an engineering context, 12th Internat. Sympos. on Rarefied Gas Dynamics, Charlottesville, Va., July 1980.

4. C. Cercignani, Theory and application of the Boltzmann equation, Scottish Academic Press, Edinburgh, 1975.

5. H. Faure, Discrépance de suites associées à un système de numération (en dimension $s$ ), Acta Arith. 41 (1982), 337-351. 
6. J. H. Halton, On the efficiency of certain quasi-random sequences of points in evaluating multi-dimensional integrals, Numer. Math. 2 (1960), 84-90; Berichtigung, ibid., p. 196.

7. E. Hlawka and R. Mück, $A$ transformation of equidistributed sequences, Applications of Number Theory to Numerical Analysis (S. K. Zaremba, ed.), Academic Press, New York, 1972.

8. M. Krook and T. T. Wu, Exact solutions of the Boltzmann equation, Phys. Fluids 20 (1977), 1589-1595.

9. L. Kuipers and H. Niederreiter, Uniform distribution of sequences, Wiley, New York, 1974.

10. C. Lécot, $A$ direct simulation Monte Carlo scheme and uniformly distributed sequences for solving the Boltzmann equation, Computing 41 (1989), 41-57.

11. _ Low discrepancy sequences for solving the Boltzmann equation, J. Comput. Appl. Math. 25 (1989), 237-249.

12. _ An algorithm for generating low discrepancy sequences on vector computers, Parallel Comput. 11 (1989), 113-116.

13. The NAG Fortran Library Manual-Mark 12, The Numerical Algorithms Group Limited, Oxford, 1987.

14. K. Nanbu, Direct simulation scheme derived from the Boltzmann equation. I, Monocomponent gases, J. Phys. Soc. Japan 49 (1980), 2042-2049.

15. H. Niederreiter and J. M. Wills, Diskrepanz und Distanz von Massen bezüglich konvexer und Jordanscher Mengen, Math. Z. 144 (1975), 125-134; Berichtigung, ibid., 148 (1976), 99.

16. H. Niederreiter, Quasi-Monte Carlo methods and pseudo-random numbers, Bull. Amer. Math. Soc. 84 (1978), 957-1041.

17. __ Point sets and sequences with small discrepancy, Mh. Math. 104 (1987), 273-337.

18. W. G. Vincenti and C. H. Kruger, Introduction to physical gas dynamics, Krieger, Malabar, 1986.

Laboratoire de Mathématiques, Université de Savoie, B.P.1104, 73011 Chambéry CedeX, France

E-mail address: lecot@frgren81.bitnet 